\title{
An Accelerative Pre-allocation Protocol for Wavelength Division Multiplexing Star-Coupled Networks
}

\author{
Chuan-Ching Sue, Sy-Yen Kuo and Yennun Huang ${ }^{\dagger}$ \\ Department of Electrical Engineering, National Taiwan University, Taipei, Taiwan \\ sykuo@cc.ee.ntu.edu.tw
}

\begin{abstract}
For the wavelength division multi-access system (WDMA), the reservation approach and the preallocation approach are two major media access protocols to support the packet-switched traffic. In this paper, a new media access control (MAC) protocol, the AP-WDMA (accelerative pre-allocation), is proposed for the WDMA. Although implemented on a simple basic architecture, it does provide a better media access protocol which can be easily extended to all the WDMA's. Through evaluations, the AP-WDMA is shown to be able to overcome the wavelength limitation through a channel sharing mechanism and enable efficient transmission with the accelerative mechanism. The APWDMA relieves these technology constraints by restricting the tunability to only one end and the table size to only $n+2$ memory spaces, where $n$ is the number of stations.
\end{abstract}

\section{Introduction}

During the past decades, the optical technology has been widely used in the high-speed long distance communications and local area networks (LAN's) [1-3]. For general applications, the wavelength division multiplexing (WDM) [4-11] is the most popular and efficient technology investigated so far to efficiently utilize the very high data rate and the vast bandwidth provided by an optical fiber.

The reservation approach and the pre-allocation approach are two main media access protocols developed for this purpose [12-17]. The P-WDMA has lower implementation and operation complexity; in addition, the R-WDMA has poor performance due to a large number of collisions and retransmissions when many stations are requesting the same data or control channels. Although several approaches were presented to improve the performance of the R-WDMA $[11,18]$ and overcome the above problem, they only illustrate the application of the protocols on specific WDM architectures with

†Acknowledgement: This research was supported by the National Science Council, Taiwan, R. O. C., under the Grant NSC 87-2213-E259-007.

Yennun Huang is with Bell Laboratories, Lucent Technologies, Inc. constraints such as tunability and large tables. The P. WDMA is not always efficient. With this method, first if the traffic is not uniform across all source-destination pairs, some stations and channels may remain idle all the time while others are waiting for the right time slots and result in larger delays. Second, if the source wants to transmit the data just after the pre-scheduled time slot that it can communicate with the destination, it has to wait for the next pre-scheduled time slot for transmission. In both cases, we will have the critical drawbacks of lower channel utilization, lower throughput, and higher delay.

Although several P-WDMA protocols were proposed with optimal performance [21-22] under the consideration of tuning time, processing time or propagation delay, they all assume that the traffic load is fixed so that they can schedule well according to the fixed load information. In realistic situations, the traffic load switches between uniform and non-uniform or high and low.

Thus a low complexity protocol is required to adjust itself to the change of traffic load. In this paper, a novel multi-access phonic protocol, AP-WDMA (accelerative pre-allocation wavelength division multi-access system), is proposed for the WDMA. It provides a better media access protocol that is based on the P-WDMA but can eliminate its drawbacks and improve the system performance with least cost. The protocol enables the source station to possibly transmit the data earlier than the pre-scheduled time slot in the above two cases through the known transmission information and the additional control channel. Even if earlier transmission can not be achieved, the AP-WDMA still works well as compared with P-WDMA. Thus, it retains the advantages of P-WDMA and improves the performance of P-WDMA. Furthermore, through channel sharing mechanisms, there is no theoretically upper limit on the maximum number of stations that can be added given a fixed number of channels. The channel sharing mechanism is similar to the mechanism proposed in [6] with the control packet transmission in AP-WDMA.

The rest of this paper is organized as follows. In Section 2, we define the architecture considered in this paper and the terminology. The novel media access 
protocol, AP-WDMA, is proposed in Section 3. Section 4 depicts the performance evaluation. Finally, we conclude our study in Section 5.

\section{Preliminaries}

In this section, we will illustrate the foundations for the AP-WDMA. Subsections 2.1 and 2.2 give the architecture definition and the terminology, respectively.

\subsection{Architecture}

The basic architecture is defined as the Fixed Transmission Tunable Reception Passive Star Architecture (FTTR-S) which is a single-hop broadcastand-select photonic network built with a passive star coupler. Fig. 1 depicts an example architecture of the FTTR-S which contains many users per station. The architecture at each station is shown in Fig. 2. The F-Tx represents the fixed transmitter, and the F-Rx and the T$\mathrm{Rx}$ represent the fixed and the tunable receivers, respectively.

Meanwhile, let $\lambda_{0}$ represent the control channel which is used for control and $A C K$ packets, and each $\lambda_{i}, 1 \leq i \leq g$, represents a data channel which is used for data transmission. $\lambda_{0}, \lambda_{1}, \lambda_{2}, \ldots, \lambda_{\mathrm{g}}$ are the available wavelengths in the system. The passive star coupler connects $n$ stations in the system. Let $g \leq n$ to accommodate the technology constraints in current devices. Each station is numbered as $1,2, \ldots, n$. Each $\lambda_{i}$ is assigned to a group of stations as a transmitting home channel. Therefore, a group of stations is identified as $S_{i}$ $=\left\{k: \lambda(k)=\lambda_{i}\right\}$ while $\lambda_{i}$ is shared among the stations. For the transmission function, each station in $S_{i}$ is provided with two fixed-tuned transmitters to avoid frequent tuning. One is fixed at $\lambda_{0}$ for controlling accelerative transmission and the other is fixed at its home channel $\lambda_{i}$ for data transmission. For the reception function, each station in $S_{i}$ is equipped with one fix-tuned and one tunable receivers to avoid frequent tuning. The fix-tuned receiver is also fixed at $\lambda_{0}$ to receive the control and $A C K$ messages while the tunable receiver can be switched between all data channels from $\lambda_{1}$ to $\lambda_{g}$ for the corresponding data reception.

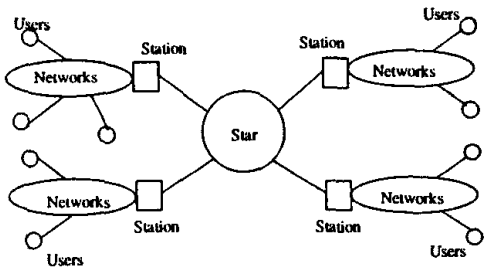

Fig. 1: Example FTTR-S with many users per station.

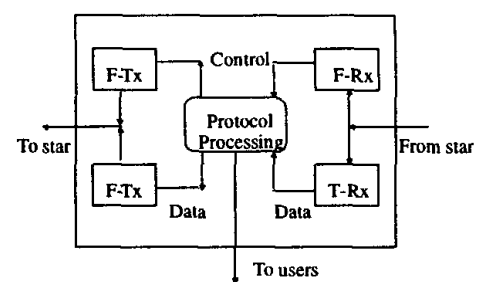

Fig. 2: The architecture at each station.

\subsection{Terminology}

In order to make the concept of the AP-WDMA clearer and to make the paper more readable, some definitions and assumptions are described as follows.

- data packet: the packet on $\lambda_{i}, 1 \leq i \leq g$, to transmit data as shown in Fig. 3. All transmitted data will be organized as one or several data packets, but they are transmitted by only one packet per data slot through the corresponding $\lambda_{i}$. Each data packet has no source id\# and destination id\#.

- control packet: the packet on $\lambda_{0}$ to transmit the control message as shown in Fig. 3. This message is used to request a possible early transmission for acceleration or normal pre-allocation transmission. Every control packet is of the same size and consists of only two fields. The first field is the protocol header, and the other field is the destination id\# whose size is only $\left\lceil\log _{2} N\right\rceil$ bits.

- ACK packet: the packet on $\lambda_{0}$ but is used to transmit the $A C K$ message as shown in Fig. 3. It is used to acknowledge the request by the control packet. It also contains two fields: the protocol header and the source id\#, which results from the destination id\# in the corresponding control packet. It has the same size with the control packet.

- data slot, $S T_{d}$ : The number of time units on $\lambda_{i}$, defined as the time of transmitting a maximum size data packet from the source station to the destination station as shown in Fig. 3. It includes the operation time for the destination $j$ to switch its tunable receiver to the corresponding $\lambda_{i}$. Besides, all data packets are assumed to require the same data slot.

- control $(A C K)$ slot: The number of time units on $\lambda_{0}$, defined as the time of transmitting the control $(A C K)$ packet from the source (destination) station to the destination (source) station as shown in Fig. 3. All control $(A C K)$ packets also require the same control slot.

- data (control, $A C K$ ) cycle: From the beginning, every $n$ data (control, $A C K$ ) slots are organized as a data (control, $A C K$ ) cycle. The control $(A C K)$ cycle time is denoted as $S T_{c}$. 
- transmission pair, $\mathrm{T}\left(i_{x}, j\right)$ : At a data slot, the data transmission from $x \in S_{i}$ to $j$ through $\lambda_{i}$ is performed, where $1 \leq i \leq g$ and $1 \leq j \leq n . \quad \mathrm{T}\left(i_{x}, j\right)$ is always initialized by the source station $x$ and determined by $j$. Even if the destination $j \in S_{k}$ tries to receive data from $x$, it is also modeled as that $\mathbf{T}\left(k_{j}, x\right)$ should first be performed by $j$ to transmit the request as the data packet.

- pre-scheduled pair, $\mathrm{P}\left(i_{x}, j\right)$ : At a data slot, the $\mathrm{T}(i, j)$ is pre-scheduled. Each node in $S_{i}$ has the opportunity to transmit its data. If $\exists i \in S_{i}$ that has data for $j, j$ arbitrates them, selects one $(x)$ from them, acknowledges its selection to $x$, then $x$ transmits; else each $i \in S_{i}$ remains idle on $\lambda_{i}$.

- early pair, $\mathrm{E}\left(i_{x}, j\right)$ : On $\lambda_{i}$, the $\mathrm{T}\left(i_{x}, j\right)$ occurs at a data slot earlier than the pre-scheduled slot of $\mathrm{P}\left(i_{x}, j\right)$ in the pre-scheduled information.

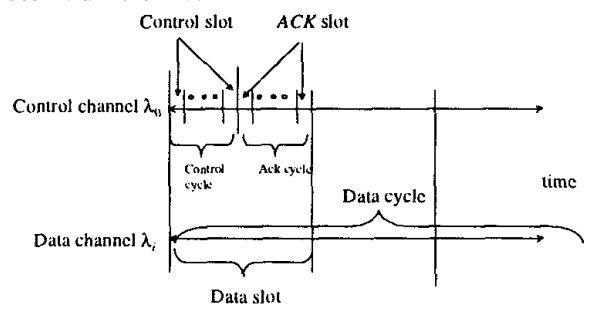

Fig. 3: Control slot (cycle), ack slot (cycle), and data slot (cycle).

\section{Media access protocol}

In this section, a new media access protocol for the AP-WDMA is proposed.

procedure PROTOCOL;

begin

Initial_condition( );

for every data slot do

begin

\{simultaneously operates on every station $i$ whose

corresponding home channel is $\lambda(i)\}$

if $\mathrm{E}\left(\lambda(i)_{i}, y\right)$ exists then perform $\mathrm{E}\left(\lambda(i)_{i}, y\right)$

else if $\mathrm{P}\left(\lambda(i)_{i, j}\right)$ exists then perform $\mathrm{P}\left(\lambda\left(i_{i}, j\right)\right.$

else remain idle;

\{each source $i$ always has a transmitter fixed on $\lambda_{0}$ \}

Control_cycle( );

\{each destination $j$ always has a receiver fixed on $\lambda_{0}$ \}

if no control packet in Control_cycle() then terminate PROTOCOL

\{no more $\mathrm{P}(i, j)$ will perform in the next $n$-1 data slots\}

else ACK_cycle( );

\{confirm possible $\mathrm{E}\left(\lambda(i)_{i}, y\right)$, normally arbitrated $\mathrm{P}\left(\lambda(i)_{i}, j\right)$ or idle for each station $i$ at the next data slot $\}$

$$
\text { end }
$$

end

Fig. 4: The media access protocol.
Our protocol is efficient for the general case where the traffic load is dynamic. Fig. 4 illustrates the protocol algorithm which will be detailed in the following three subsections. Subsection 3.1 discusses the operations on each station $i$, including the time-interleaved prescheduling. Subsection 3.2 illustrates the control packet transmission. Then the $A C K$ packet transmission and the confirmation of the early pair is depicted in Subsection 3.3.

\subsection{Operations on each station}

In the beginning, the protocol performs the procedure Initial_condition( ) for each station $i$ to restart the channel utilization from the beginning of the prescheduled table. The configuration of the network, e.g. group information, is established at this time. Then for every data slot, simultaneously on each station $i$, one of the following cases will occur. 1) If $\mathrm{E}\left(\lambda(i)_{i}, y\right)$ exists, then perform $\mathrm{E}\left(\lambda(i)_{i}, y\right)$ because no other pre-scheduled $\mathrm{P}\left(\lambda(i)_{i}, j\right), \quad 1 \leq i \leq g, \quad 1 \leq j \leq n$, can perform earlier than $\mathrm{P}\left(\lambda(i)_{i}, y\right)$ by the $\mathrm{E}\left(\lambda(i)_{i}, y\right)$ construction rules in Subsections B and C. 2) If $\mathrm{E}\left(\lambda(i)_{i}, y\right)$ does not exist, then perform $\mathrm{P}\left(\lambda(i)_{i}, j\right)$ when station $i$ is arbitrated to have the data for station $j$, or will remain idle otherwise.

In Table 1, this pre-scheduled information is established based on the time-channel-receiver station relationship. It illustrates that, for every data cycle, each $\lambda_{i}$ is used as the receiving wavelength one by one by each station. And the usage of the table is that, for every data cycle, only the destination $j$ can receive the data from the source $S_{i}$ on $\lambda_{i}$ at $T=t_{h}$ with $j=(i+h-I) \bmod n$. In the equation, $j=0$ means $j=n$.

For the AP-WDMA, each data packet doesn't have to indicate its source address bits and the destination address because the coordination is done in the control channel. The address is indicated by the authorized station, which can transmit and receive on this channel at each data slot in every data cycle. In addition, a station does not have to know all the information in the entire table to correctly execute the protocol. Instead, only the following two points have to be known by each station. 1) For data transmission, each source node in $S_{i}$ must know the corresponding destination id\# at each data slot (on $\lambda_{i}$ actually). $S_{i}$ will store the formula in the memory space, which is used to derive id\# identical to the information appeared in the corresponding row of $\lambda_{i}$ in Table 1. Therefore, in every data cycle, the id\# of the destination $j$ at $\mathrm{T}=t_{h}$ is derived from the formula that should be the same as the element in the $h$-th member of the corresponding row. 2) For data reception, each destination $j$ must know the corresponding home channel 
id\# of the source at each data slot. The destination $j$ has to know these information in the $\operatorname{Ar}(j)$ array which is shown in Table 2 based on the time- $\operatorname{Ar}(j)$-group relationship. Therefore, in every data cycle, the home channel id\# of source $S_{i}$ at $T=t_{h}$ which appears in the $h$ th member of $\operatorname{Ar}(j)$ is derived with the formula of $i=(j-$ $h+1) \bmod n$. If $i=0, g+1, g+2, \ldots, n$, the corresponding element is kept empty and the station knows that no prescheduling is in this data slot.

Table 1: The pre-scheduled information based on the timechannel-receiver station relationship.

\begin{tabular}{|c|c|c|c|c|c|}
\hline Channel/Time & $t_{1}$ & $t_{2}$ & $\cdots$ & $t_{n-1}$ & $t_{n}$ \\
\hline$\lambda_{1}$ & 1 & 2 & $\cdots$ & $n-1$ & $n$ \\
\hline$\lambda_{2}$ & 2 & 3 & $\ldots$ & $n$ & 1 \\
\hline$\lambda_{3}$ & 3 & 4 & $\ldots$ & 1 & 2 \\
\hline$\cdots$ & $\cdots$ & $\cdots$ & $\cdots$ & $\cdots$ & $\cdots$ \\
\hline$\overline{\lambda_{i}}$ & $i$ & $i+1$ & $\cdots$ & $(n-2+i)_{n}$ & $(n-1+I)_{n}$ \\
\hline$\cdots$ & $\cdots$ & $\cdots$ & $\cdots$ & $\cdots$ & $\cdots$ \\
\hline$\lambda_{\mathrm{g}}$ & $(g)_{n}$ & $(g+1)_{n}$ & $\cdots$ & $(n-1+g)_{n}$ & $(n+g)_{n}$ \\
\hline
\end{tabular}

$(\mathrm{X})_{n}=\mathrm{X} \bmod n$

\subsection{Control packet transmission}

While data are being transmitted, simultaneously on $\lambda_{0}$ at every data slot, the procedure Control_cycle( ) is performed by each node for the control packet transmission. It is based on the following two policies. 1) The time-interleaved pre-scheduling is used such that, in every control cycle, the $i$-th control slot is only for station $i$ to transmit the control packet. 2) At the $i$-th control slot, whether station $i$ transmits the control packet or not is decided by the check-and-send policy. Here, the first rule is to prevent collisions. This is because there is only one $\lambda_{0}$ for all stations to transmit the control packets. More than one station transmitting control packets at the same control slot must be avoided even though these control packets are between different source and destination stations. Therefore, each data slot is defined to contain one control and one $A C K$ cycles where every control $(A C K)$ slot is dedicated to only one corresponding station with the time-interleaved scheduling. Meanwhile, the check-and-send policy decides the behavior of control packet transmission at the $i$-th control slot with one of the following three cases: 1) If station $i$ has no data for any other station after this data slot, i.e., no other $\mathrm{P}\left(\lambda(i)_{i}, j\right)$ for $1 \leq j \leq n$ will be performed during the next $n-1$ data slots, then station $i$ remains idle. 2) If the next possible $\mathrm{T}\left(\lambda(i)_{i}, j\right)$ for station $i$ is $\mathrm{P}\left(\lambda(i)_{i}, j\right)$ at the next data slot, then station $i$ transmits the control packet containing the id\# of station $j$ as the address bits. 3) Otherwise, an early transmission for acceleration may be possible at the next data slot. Station $i$ will transmit the control packet whose address bits indicate the destination id\# of the next possible $\mathrm{E}\left(\lambda(i)_{i}, y\right)$. But, each control packet is without the source address because the $i$-th control slot already indicates station $i$ as the source station. In addition, no other control message except the destination address is needed in each control packet.

Table 2: The reception information array $\operatorname{Ar}(j)$ in each station.

\begin{tabular}{|c|c|c|c|c|c|c|}
\hline $\operatorname{Ar}(\mathrm{j}) / \mathrm{T}$ & $t_{l}$ & $t_{2}$ & $T_{3}$ & $\cdots$ & $t_{n-1}$ & $t_{n}$ \\
\hline $\operatorname{Ar}(1)$ & 1 & Empty & $\cdots$ & $\cdots$ & 3 & 2 \\
\hline $\operatorname{Ar}(2)$ & 2 & 1 & Empty & $\cdots$ & 4 & 3 \\
\hline$\cdots$ & $\cdots$ & $\cdots$ & $\cdots$ & $\cdots$ & $\cdots$ & $\cdots$ \\
\hline $\operatorname{Ar}(i)$ & $i$ & $i-1$ & $i-2$ & & $(i+2)_{n}$ & $(i+1)_{n}$ \\
\hline$\cdots$ & $\cdots$ & $\cdots$ & $\cdots$ & $\cdots$ & $\cdots$ & $\cdots$ \\
\hline $\operatorname{Ar}(n)$ & $\cdots$ & $\cdots$ & $\cdots$ & $\cdots$ & 2 & 1 \\
\hline
\end{tabular}

\subsection{ACK packet transmission}

After the Control_cycle( ), the protocol performs the following steps to construct the transmission pair which is either Early pair or Pre-scheduled pair. 1) Each destination $j$ always has a receiver fixed at $\lambda_{0}$. If station $j$ finds no control packet during this control cycle (actually all stations should have the same information), it indicates that there will be no transmission in the next $n$ 1 data slots. No $A C K$ packet is transmitted by station $j$ (actually no $A C K$ packet is transmitted by any station). The protocol can be terminated at the next data slot and will be restarted under another system request. 2) Else, the procedure ACK_cycle( ) is performed by each station $j$. In the first part of ACK_cycle( ), the source id\# of the possible transmission pair is decided by the check-anddiscard policy and $n$ corresponding memory spaces. A random number generator is needed for the receiver in each node to arbitrate among more than one certified node. All the received control packets are checked one by one as they are transmitted in Control_cycle( ). The processing steps are illustrated as follows. a) For each control packet, the source id\# (represented as $f$ ) of the first control packet destined to each station is stored in the corresponding memory space. Therefore, $n$ memory spaces are reserved. b) The source id\# (represented as $x$ ) of each following control packet destined to the same 
station is compared with $f$. If $x$ and $f$ have the same home channel, randomly select $x$ or $f$. Else if $\mathrm{P}\left(\lambda(x)_{x}, j\right)$ is earlier than $\mathrm{P}\left(\lambda\left(f_{f}, j\right)\right.$ in the next $n-1$ data slots $(\operatorname{Ar}(j)$ can be referenced), then $x$ replaces $f$ in the memory space. Otherwise, the new incoming control packet is discarded. c) Therefore, after one control cycle, each memory space has either a source id\# or nothing. Each one forms a transmission pair and has a corresponding pre-scheduled time slot. Sequentially compare the pre-scheduled time slots which have the same home channel id\# as that of the source $\mathrm{id \#}$ in the memory space $j$ and determine the final unique transmission pair. Transmission pair can be either $\mathrm{P}\left(\lambda(i)_{i}, j\right)$ or $\mathrm{E}\left(\lambda(i)_{i}, j\right)$.

After the above decision has been made, the second part of ACK_cycle( ) is for $A C K$ packet transmission decided by the following policies. a) The timeinterleaved pre-scheduling is used such that the $j$-th $A C K$ slot of each $A C K$ cycle is pre-scheduled for station $j$ to transmit the $A C K$ packet. This is also to prevent collisions with a reason similar to that in Control_cycle( ). b) At the $j$-th $A C K$ slot, whether station $j$ transmits the $A C K$ packet or not is decided by the check-and-send policy with the following steps. i) If station $j$ has no $A C K$ for any other station during this $A C K$ cycle, station $j$ remains idle. ii) Otherwise, station $j$ transmits the unique $A C K$ packet with $i$ as the destination address bits to confirm $\mathbf{T}\left(\lambda(i)_{i}, j\right)$. Hence each source station which has transmitted the control packet destined to station $j$ in Control_cycle( ) need only look into the $j$ th $A C K$ slot in the $A C K$ cycle. And the selected source $i$ will need to perform $\mathrm{T}\left(\lambda(i)_{i}, j\right)$ at the next data slot. In this manner, the processing complexity is reduced. No more $A C K$ message including the source address is needed in each $A C K$ packet because the $j$-th $A C K$ slot already indicates station $j$ as the source station. In addition, while transmitting the $A C K$ packet, station $j$ can tune its receiver to the corresponding channel $\lambda(i)$ to reduce the tuning latency. The data packet of $\mathrm{T}\left(\lambda(i)_{i}, j\right)$ also needs no address id\#. Although in fact only $g A C K$ slots are used since only $g$ available channels can make concurrent transmissions, $n A C K$ slots are used for low operation complexity.

\section{Evaluations}

This section evaluates the performance of the APWDMA. We use probability analysis method to evaluate the impact of the early pair.

\subsection{Probability analysis}

This subsection quantitatively evaluates the occurrence probability of each behavior case at a data slot on a given $\lambda_{i}$. Since only the beginning data slot enables no early transmission, without loss of generality, the first data slot is neglected from this evaluation consideration. Furthermore, because we focus on the early transmission effect instead of the channel sharing effect, only the case that the number of channels is equal to the number of stations is analyzed for simplicity. First, let $G$ be the arrival rate of data packets generated by each station per data slot that is modeled by the Poisson process. The balanced traffic is assumed and analyzed. Thus, $G$ is the same across all the stations. The probability of $k$ data packets generated per station per data slot is then: $p(k)=$ $e^{-G} G^{k} / k$ !. In the AP-WDMA, the behavior on any channel at a given data slot has only two states: idle or generating a packet. Therefore, the probability conditions become the followings [17]:

$$
\begin{aligned}
& p(0)=e^{-G} \text {, no data packet is generated at a data slot. } \\
& p(1)=1-e^{-G} \text {, only one data packet is generated at }
\end{aligned}
$$
this data slot.

$p(k)=0, k>1$, more than one packet is generated at this data slot.

However, the packet generated at any data slot on a given $\lambda_{i}$ can destine to only one out of the $n$ stations (multicasting is prohibited). For convenience, let the receiving stations be listed as $\mathrm{A}_{0}, \mathrm{~A}_{1}, \ldots, \mathrm{A}_{n-I}$ where each $\mathrm{A}_{h}, 0 \leq h \leq n-1$, represents the receiving station which will enable the corresponding $\mathrm{T}(i, j)$ to be an $\mathrm{E}(i, j)$ with $h$ slots earlier. (The $\operatorname{Ar}(j)$ array can be referenced and $h=$ 0 means the corresponding $\mathrm{T}(i, j)$ is the $\mathrm{P}(i, j)$ at this slot.) Under the above conditions, let $r(h)$ represent the probability fraction of each corresponding case. Therefore, $r(0)=1 / n$ because the packet is exactly received by $\mathrm{A}_{0}$, i.e., only one out of $N$ stations can correctly receive the packet on this $\lambda_{i} . \quad r(1)=$ $[1 / n][p(0)+p(1)(n-1) / n]$ because the packet is exactly received by $A_{1}$ and $A_{1}$ can not receive any data on its prescheduled channel (differs from $\lambda_{i}$ but has the same packet generation model), which includes 2 cases: 1 ) this pre-scheduled channel is idle; 2 ) it has a packet but is not for $\mathrm{A}_{1}$. And $r(h)=[1 / n][p(0)+p(1)(n-1) / n]\left(^{n-1} C_{h-1}\right.$ $\left.[p(1) /(n)]^{h-1}(h-1) !\right\}$ because: 1$)$ the packet is exactly received by $A_{h}$; 2) $A_{h}$ does not receive at its prescheduled channel; 3) each $A_{1}$ to $A_{h-1}$ is busy in its individual data reception, i.e., at least $h$ - 1 out of other $n$ 1 channels are generating the packets which are received by these $h-1$ stations on one-to-one basis.

With the media access protocol in Section III, each $A_{k}$ already switches the receiver to receive the data before this packet is generated for $A_{k}$ such that the value of each $s(k), 1 \leq k \leq n-1$, can be modeled as 0 . In addition, both the P-WDMA and the AP-WDMA have the same $p(0)$ 
value. Therefore, meaningful comparisons of probabilities should be only between each $R(h)=$ $p(1) r(h) / K$, with $0 \leq h \leq n-1$ and $K=r(0)+r(1)+\ldots+$ $r(n-1)$, where each $R(h)$ represents the occurrence probability of corresponding $\mathrm{T}(i, j)$ with $h$ slots earlier. These comparisons are illustrated in the following figures under different bases.

In Fig. 5, with $G$ as the parameter, the relationship between $R(h)$ and $h$ is illustrated at $n=20$. As expected, it reveals that almost all $R(h)$ curves decrease as $h$ increases because it is harder to achieve more early slots. Besides, $R(h)_{G=0.1}<R(h)_{G=0.5}<R(h)_{G=1}<R(h)_{G=2}<$ $R(h)_{G=5}$ for most $h$ values because $p(1)$ and each $R(h)$ increase as $G$ increases. Fig. 6 depicts the relationship between $R(h)$ and $h$ at $G=2$. It also illustrates that all three $R(h)$ curves decrease as $h$ increases for the similar reason in Fig. 6. However, for small $h, R(h)_{n=1000}<$ $R(h)_{n=100}<R(h)_{n=10}$ because too many stations prevent $\mathrm{A}_{h}$ from achieving early transmissions. But for large $h$, $R(h)_{n=10}<R(h)_{n=100}<R(h)_{n=1000}$ because the sum of all $R(h)$ 's equals $p(1)$ for each $n$.

In addition, the system load (represented as $d=$ $p(1))$ has been used as a parameter in Fig. 7 and Fig. 8 to clearly indicate each $R(h)$ probability under different system loads. Based on this derivation, Fig. 7 illustrates the relationship between $R(h)$ and $h$ at $n=20$. As expected, it reveals that all three $R(h)$ curves decrease as $h$ increases and $R(h)_{d=0.1}<R(h)_{d=0.3}<R(h)_{d=0.5}<R(h)_{d=0.7}$ $<R(h)_{d=1}$ with similar reasons as those in Fig. 5. In Fig. 8 , the relationship between $R(h)$ and $h$ is depicted at $d=$ 0.5 . It also illustrates that, with similar reasons as in Fig. 6, all three $R(h)$ curves decrease as $h$ increases as well as $R(h)_{n=1000}<R(h)_{n=100}<R(h)_{n=10}$ for small $h$ and $R(h)_{n=10}$ $<R(h)_{n=100}<R(h)_{n=1000}$ for large $h$. Therefore, all the above results reveal that early transmissions are possible and the AP-WDMA is useful in the balanced traffic whether the load is high or low. As for the unbalanced traffic load, the third case in the derivation of $r(h)$ can be higher like the example $T(2,1)$ transmission provided in the $\lambda_{2}$ of Table 5 . When the traffic locality occurs in $\mathrm{T}(2,1)$, the early transmission can make the delay shorter. The early transmission provided in AP-WDMA can occur in various traffic loads from balanced to unbalanced or high to low. Due to the space limitation, the probability evaluation of unbalanced traffic load and channel sharing mechanism is left as future work.

\section{Conclusions}

We have proposed a new multiaccess photonic model, the AP-WDMA/early transmission. AP-WDMA is based on the P-WDMA without its drawbacks and improves the system performance with the least cost.
The technique is to allow the source station to possibly transmit the data earlier than the pre-scheduled time slot in the P-WDMA through the known-ahead transmission information and the additional control channel. Through probability evaluation on the AP-WDMA, it was shown that the AP-WDMA does reduce delays in the P-WDMA and improve the system performance significantly for balanced traffic load. Even if earlier transmission can not be performed, the AP-WDMA will still work just like a P-WDMA and retain its advantages. Furthermore, the channel sharing and time interleaving mechanism in the AP-WDMA can easily support more nodes than available channels. In addition, the protocol works well under different traffic loads because it can automatically reduce delays by the accelerative transmission.

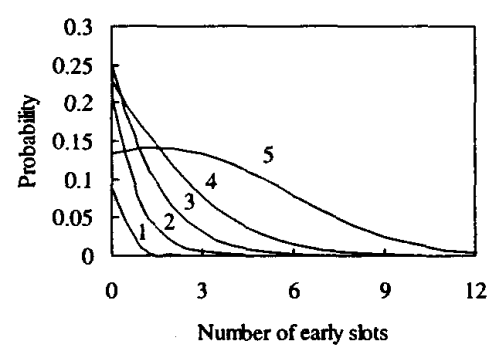

Fig. 5: $R(h)$ vs. $h$ at $n=20$, with $G$ as the parameter. Curves 1 to 5 are for $G=0.1,0.5,1,2$, and 5 , respectively.

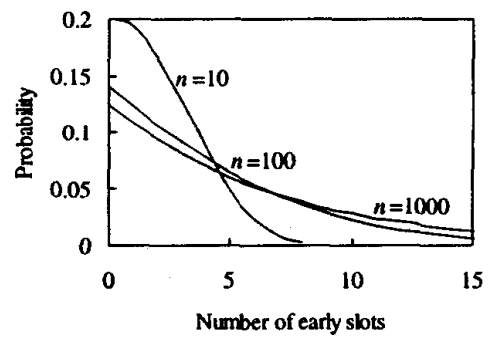

Fig. 6: $R(h)$ vs. $h$ at $G=2$, with $n$ as the parameter.

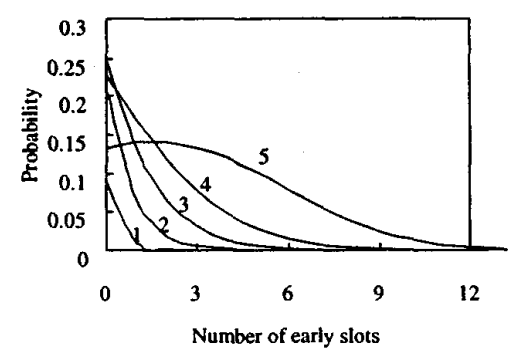

Fig. 7: $R(h)$ vs. $h$ at $n=20$, with $d$ as the parameter. Curves 1 to 5 are for $d=0.1,0.3,0.5,0.7$, and 1 , respectively. 


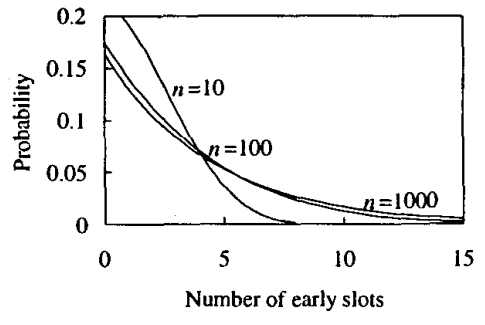

Fig. 8: $R(h)$ vs. $h$ at $d=0.8$, with $d$ as the parameter.

\section{References}

[1] C. A. Brackett, A. S. Acampora, J. Sweitzer, et al., "A Scalable Multiwavelength Multihop Optical Network: A Proposal for Research on All-Optical Networks," IEEE J. Lightwave Tech., vol. 11, no. 5/6, pp. 736-753, May/June, 1993.

[2] P. E. Green, L. A. Coldren, K. M. Johnson, et al., "AllOptical Packet-Switched Metropolitan-Area Network Proposal," IEEE J. Lightwave Tech., vol. 11, no. 5/6, pp. 754-763, May/June, 1993.

[3] C. A. Brackett, "Dense Wavelength Division Multiplexing Networks: Principles and Applications," IEEE J. Select. Areas Commu., vol. 8, no. 6, pp. 948-964, August 1990.

[4] A. Ganz and Y. Gao, "Time-Wavelength Assignment Algorithms for High Performance WDM Star Based Systems," IEEE Trans. on Commu., vol. 42, no. 2/3/4, pp. 1827-1836, Feb./Mar./Apr. 1994.

[5] S. T. Tan and D. Du, "Embedded Unidirectional Incomplete Hypercubes for Optical Networks," IEEE Trans. on Commu., vol. 41, no. 9, pp. 1284-1289, Sept. 1993.

[6] K. A. Williams, T. Q. Dam, and D. Du, "A Media-Access Protocol for Time and Wavelength Division Multiplexed Passive Star Networks," IEEE J. Select. Areas Commu., vol. 11, no. 4, pp. 560-567, May 1993.

[7] J. Sasaki and N. Takato, "An Optical Multiaccessor Designed for a Fiber-Optic Star-Ring LAN," IEEE $J$. Lightwave Tech., vol. 10, no. 2, pp. 250-254, Feb. 1992.

[8] M. G. Hiuchyj and M. J. Karol, "ShuffleNet: An Application of Generalized Perfect Shuffles to Multihop Lightwave Networks," IEEE J. Lightwave Tech., vol. 9, no. 10, pp. 1386-1397, Oct. 1991.

[9] G. N. Sudhakar, N. D. Georganas, and M. Kavehrad, "Slotted Aloha and Reservation Aloha Protocols for Very High-Speed Optical Fiber Local Area Networks Using Passive Star Topology," IEEE J. Lightwave Tech., vol. 9, no. 10, pp. 1411-1422, Oct. 1991.

[10] M. S. Goodman, H. Kobrinski, M. P. Vecchi, et al., "The LAMBDANET Multiwavelength Network: Architecture, Applications, and Demonstrations," IEEE J. Select. Areas Commu., vol. 8, no. 6, pp. 995-1004, August 1990.

[11] M. S. Chen, N. R. Dono, and R. Ramaswami, "A MediaAccess Protocol for Packet-Switched Wavelength
Division Multiaccess Metropolitan Area Networks," IEEE J. Select. Areas Commu., vol. 8, no. 6, pp. 10481057, August 1990.

[12] P. W. Dowd, K. Bogineni, K. A. Aly, J. A. Perreault, et al., "Hierarchical scalable photonic architectures for highperformance processor interconnection," IEEE Trans. on Computers, vol. 42, no. 9, pp. 1105-1120, Sept. 1993.

[13] K. Bogineni, K. M. Sivalingam, and P. W. Dowd, "Low Complexity Multiple Access Protocols for WavelengthDivision Multiplexed Photonic Networks," IEEE J. Select. Areas Commu., vol. 11, no. 4., pp. 590-604, May 1993.

[14] K. Bogineni and P. W. Dowd, "A collisionless multiple access protocol for a wavelength division multiplexed star-coupled configuration: architecture and performance analysis," IEEE J. Lightwave Tech., vol. 10, no. 11, pp. 1688-1699, Nov. 1992.

[15] P. W. Dowd, "Wavelength division multiple access channel hypercube processor interconnection," IEEE Trans. on Computers, vol. 41, no. 10, pp. 1223-1241, Oct. 1992.

[16] P. W. Dowd, "Random access protocols for high speed interprocessor communication based on a passive star topology," IEEE J. Lightwave Tech., vol. 9, no. 6, pp. 799-808, June 1991.

[17] Chen-Ken Ko and Sy-Yen Kuo, "Multiaccess Processor Interconnection Using Subcarrier and Wavelength Division Multiplexing," to be published in IEEE $J$. Lightwave Tech., Feb. 1997.

[18] C. J. Hou, B. Wang, and C. C. Han, "Design and Analysis of a WDMA Protocol for Passive Star-Coupled Lightwave Networks," Infocom 96, pp. 1225-1233, Feb. 1996.

[19] J. P. Weber, B. Stoltz, H. Sano, et. al., "An Integratable Polarization-Independent Tunable Filter for WDM Systems: The Multigrating Filter," IEEE J. Lightwave Tech., vol. 14, no. 12, pp. 2719-2756, Dec. 1996.

[20] E. L. Wooten, R. L. Stone, E. W. Miles, and E. M. Bradley, "Rapidly Tunable Narrowband Wavelength Filter Using LiNbO3 Unbalanced Mach-Zehnder Interferometer," IEEE J. Lightwave Tech., vol. 14, no. 11, pp. 2530-2536, Nov. 1996.

[21] G. N. Rouskas and M. H. Ammar, "Analysis and Optimization of Transmission Schedules for Single-Hop WDM Networks," IEEE/ACM Tran. on Networking, vol. 3, no. 2, pp. 211-221, Apr. 1995.

[22] F. Jia, B. Mukherjee, and J. Iness, "Scheduling VariableLength Messages in a Single-Hop Multichannel Local Lightwave Network," IEEE/ACM Tran. on Networking, vol. 3, no. 4, pp. 477-488, Aug. 1995.

[23] P. A. Humblet, R. Ramaswami, and K. N. Sivarajan, "An Efficient Communication Protocol for High-Speed PacketSwitched Multichannel Networks," IEEE J. Select. Areas Commun., vol. 11, no. 4, pp. 568-578, May 1993.

[24] D. Guo, Y. Yemini and Z. Zhang, "Scalable High-Speed Protocols for WDM Optical Star Networks," IEEE Globecom '94, pp. 1544-1551, Dec. 1994. 